ИЗВЕСТИЯ АКАДЕМИИ НАУК ЭСТОНСКОН ССР. ТОМ 28 ФИЗИКА * МАТЕМАТИКА. 1979, № 3

\title{
К ТЕОРИИ СИНТЕЗА РАВНОСЛОЙЫХ ДИЭЛЕКТРИЧЕСКИХ ИНТЕРФЕРЕНЦИОННЫХ ПЛЕНОК
}

\begin{abstract}
Введение
Под синтезом многослойной интерференционной пленки понимается нахождение ее состава по заданной спектральной характеристике. Долгое время эта задача не имела общего решения. Лишь недавно в $\left[{ }^{1,2}\right]$ были заложены основы общего метода синтеза пленок и продемонстрирована его эффективность. В настоящей статье мы изложим другой метод, не столь общий, но не лишенный своих преимуществ. В качестве ограничительного условия мы примем равенство оптических толщин всех слоев пленки (условие равнослойности). Это условие, как известно, сильно упрощает задачу. Число слоев тоже задается наперед. Таким образом, синтез состоит в нахождении наиболее выгодных значений показателей преломления слоев. Поглощением мы будем, как обычно, пренебрегать.

Такая ограниченная задача синтеза уже рассматривалась во многих работах, причем был получен целый ряд конкретных решений. По изяществу подхода привлекает особое внимание статья $\left[{ }^{3}\right]$. Мы предложим здесь близкий, но более простой и прозрачный метод. Ранее аналогичный метод был использован в задаче синтеза интерференционно-поляризационных светофильтров [ $\left.{ }^{4}\right]$.
\end{abstract}

\section{Основные формулы}

В основу предстоящего теоретического синтеза диэлектрических равнослойных интерференционных пленок положим выведенные в [ $\left.{ }^{5}\right]$ (см. гл. III) общие формулы для спектральных характеристик многослойных пленок. Для величин $a=r / t$ и $b=t^{-1}$, где $r$ и $t-$ амплитудные коэффициенты отражения и пропускания, имеем два варианта формул. Приведем их в том виде, какой они принимают в случае равенства оптических толщин всех слоев. Во-первых,

- $a=r l t=\Sigma i^{s} \sin ^{s} \alpha \cos ^{N-s} \alpha \operatorname{sh}\left(v_{0}-2 v_{k_{1}}+2 v_{k_{2}}-+\ldots+(-1){ }^{s} 2 v_{k_{s}}-\right.$

$$
\left.-(-1)^{s} v_{N+1}\right) \text {, }
$$

$$
\begin{gathered}
b=t^{-1}=\sum i^{s} \sin ^{s} \alpha \cos ^{N-s} \alpha \operatorname{ch}\left(v_{0}-2 v_{k_{1}}+2 v_{k_{2}}-+\ldots\right. \\
\left.\ldots+(-1)^{s} 2 v_{k_{s}}-(-1)^{s} v_{N+1}\right)
\end{gathered}
$$

и, во-вторых,

$$
\begin{gathered}
a=r l t=\Sigma \times h v_{01} \times h v_{12} \ldots \text { xh } v_{N, N+1} \exp \left[-i \alpha\left(\varepsilon_{1}+\varepsilon_{2}+\ldots+\varepsilon_{N}\right)\right], \\
b=t^{-1}=\Sigma \times h v_{01} \times h v_{12} \ldots \text { xh } v_{N, N+1} \exp \left[i \alpha\left(\varepsilon_{1}+\varepsilon_{2}+\ldots+\varepsilon_{N}\right)\right] .
\end{gathered}
$$


Здесь $N$ - число слоев, перенумерованных в направлении падения света; индексы 0 и $N+1$ относятся к исходной среде и подложке;

$$
\alpha=k n h=(2 \pi / \lambda) n h,
$$

где $k-$ волновое число, $\lambda$ - длина волны в вакууме, $n$ и $h-$ показатель преломления и толщина любого слоя (дисперсией мы пренебрегаем); величины $v_{m}$ и $v_{m, m+1}(m=0,1, \ldots, N+1)$ определяются формулами

$$
\begin{gathered}
v_{m}=(1 / 2) \ln n_{m}, \\
v_{m, m+1}=v_{m}-v_{m+1}=(1 / 2) \ln \left(n_{m} / n_{m+1}\right) .
\end{gathered}
$$

Формулы (5)-(7) верны в таком виде при нормальном падении света, что мы и будем предполагать. Притом, так как все показатели преломления вещественны, то и $\alpha, v_{m}$ и $v_{m, m+1}$ вещественны. В формулах (1) и (2) суммы берутся по всем комбинациям $k_{1}, k_{2}, \ldots, k_{s}$, которые можно составить из индексов $1,2, \ldots, N$, причем $k_{1}<k_{2}<\ldots<k_{s}$ и $s=0,1, \ldots, N$. Число всех этих комбинаций равно $2^{N}$. В формулах (3) и (4) хh означает sh или ch, причем суммы берутся по всем возможным распределениям xh на sh и ch: в формуле (3) - по всем распределениям с нечетным числом sh, а в формуле (4) - по всем распределениям с четным числом sh. Число тех и других распределений равно $2^{N}$. Числа $\varepsilon_{1}, \varepsilon_{2}, \ldots, \varepsilon_{N}$ определяются правилом: $\varepsilon_{1}=+1$, если $\mathrm{xh}_{01}$ есть $\operatorname{ch} v_{01}$, и $\varepsilon_{1}=-1$, если хһ $v_{01}$ есть $s h v_{01} ; \varepsilon_{m} \varepsilon_{m+1}=+1$, если хh $v_{m, m+1}$

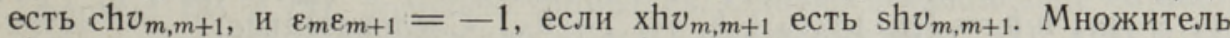
$\mathrm{xh} v_{N, N+1}$ не участвует в определении значений $\varepsilon_{1}, \varepsilon_{2}, \ldots, \varepsilon_{N}$, но он обеспечивает нечетность числа sh в каждом члене формулы (3) и четность числа sh в каждом члене формулы (4).

Формулы (1)-(4), как показано в [ $\left.{ }^{5}\right]$ (см. гл. III), вытекают из матричной формулы

$$
F=G\left(v_{0}\right) L_{1} L_{2} \ldots L_{N} G\left(-v_{N+1}\right),
$$

где

$$
L_{m}=\cos \alpha \cdot E+i \sin \alpha \cdot \bar{G}\left(-2 v_{m}\right),
$$

$E-$ единичная матрица,

$$
\begin{aligned}
& G(v)=\left(\begin{array}{ll}
\operatorname{ch} v & \operatorname{sh} v \\
\operatorname{sh} v & \operatorname{ch} v
\end{array}\right), \\
& \bar{G}(v)=\left(\begin{array}{l}
\operatorname{ch} v-\operatorname{sh} v \\
\operatorname{sh} v-\operatorname{ch} v
\end{array}\right)
\end{aligned}
$$

II

$$
F=\left(\begin{array}{ll}
b & a^{*} \\
a & b^{*}
\end{array}\right)
$$

Последняя формула верна в таком виде в случае отсутствия поглощения в средах, что мы и предполагаем.

Перейдем от характеристик $a$ и $b$ к энергетическим коэффициентам отражения и пропускания $R$ и $T$ :

$$
\begin{aligned}
& a a^{*} \equiv A=R / T, \\
& b b^{*} \equiv B=T^{-1} .
\end{aligned}
$$

Переписав формулы (3) и (4) в виде 


$$
\begin{aligned}
& a=e^{-i N \alpha}\left(a_{0}+a_{1} e^{2 i \alpha}+\ldots+a_{N} e^{2 i N \alpha}\right), \\
& b=e^{-i N \alpha}\left(b_{0}+b_{1} e^{2 i \alpha}+\ldots+b_{N} e^{2 i N \alpha}\right),
\end{aligned}
$$

где $a_{0}, a_{1}, \ldots, a_{N}$ и $b_{0}, b_{1}, \ldots, b_{N}$ вещественны и зависят определенным образом только от $v_{01}, v_{12}, \ldots, v_{N, N+1}$, находим дляя $A$ и $B$ следующие выражения:

$$
\begin{aligned}
& A=A_{0}+A_{1} \cos ^{2} \alpha+\ldots+A_{N} \cos ^{2 N} \alpha, \\
& B=B_{0}+B_{1} \cos ^{2} \alpha+\ldots+B_{N} \cos ^{2 N} \alpha .
\end{aligned}
$$

Очевидно, коэффициенты $A_{0}, A_{1}, \ldots, A_{N}$ являются однородными квадратичными функциями от $a_{0}, a_{1}, \ldots, a_{N}$, а $B_{0}, B_{1}, \ldots, B_{N}-$ функциями того же вида от $b_{0}, b_{1}, \ldots, b_{N}$. А так как

$$
b b^{*}-a a^{*}=B-A=T^{-1}-R / T=1,
$$

то эти коэффициенты связаны соотношениями

$$
\begin{gathered}
B_{0}=1+A_{0}, \\
B_{k}=A_{k}, \quad k=1,2, \ldots, N .
\end{gathered}
$$

Найдем еще несколько простых соотношений между коэффициентами $a_{k}, b_{k}, A_{k}$ и $B_{k}(k=0,1, \ldots, N)$. Положив в формулах (1) и (2) $\cos \alpha= \pm 1$, находим $a=( \pm 1)^{N} \operatorname{sh}\left(v_{0}-v_{N+1}\right)$ и $b=( \pm 1)^{N} \operatorname{ch}\left(v_{0}-v_{N+1}\right)$. С другой стороны, формулы (14) и (15) дают: $a=( \pm 1)^{N}\left(a_{0}+a_{1}+\ldots\right.$ $\left.\ldots+a_{N}\right)$ и $b=( \pm 1)^{N}\left(b_{0}+b_{1}+\ldots+b_{N}\right)$. Следовательно,

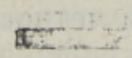

$$
\begin{aligned}
& a_{0}+a_{1}+\ldots+a_{N}=\operatorname{sh}\left(v_{0}-v_{N+1}\right), \\
& b_{0}+b_{1}+\ldots+b_{N}=\operatorname{ch}\left(v_{0}-v_{N+1}\right) .
\end{aligned}
$$

А так как при $\cos \alpha= \pm 1$, согласно формулам (16) и (17), $a a^{*}=$ $=A_{0}+A_{1}+\ldots+A_{N}$ и $b b^{*}=B_{0}+B_{1}+\ldots+B_{N}$, то

$$
\begin{aligned}
& A_{0}+A_{1}+\ldots+A_{N}=\left(a_{0}+a_{1}+\ldots+a_{N}\right)^{2}=\operatorname{sh}^{2}\left(v_{0}-v_{N+1}\right), \\
& B_{0}+B_{1}+\ldots+B_{N}=\left(b_{0}+b_{1}+\ldots+b_{N}\right)^{2}=\operatorname{ch}^{2}\left(v_{0}-v_{N+1}\right) .
\end{aligned}
$$

Эти формулы согласуются с формулами (19). Положим теперь $\sin \alpha= \pm 1$. Тогда из формул (1), (2) и (14)-(17) следует

$$
\begin{gathered}
a_{0}-a_{1}+\ldots+(-1)^{N} a_{N}= \\
=(-1)^{N} \operatorname{sh}\left(v_{0}-2 v_{1}+2 v_{2}-+\ldots+(-1)^{N} 2 v_{N}-(-1)^{N} v_{N+1}\right), \\
b_{0}-b_{1}+-\ldots+(-1)^{N} b_{N}= \\
=(-1)^{N} \operatorname{ch}\left(v_{0}-2 v_{1}+2 v_{2}-+\ldots+(-1)^{N} 2 v_{N}-(-1)^{N} v_{N+1}\right)
\end{gathered}
$$

и

$$
\begin{aligned}
A_{0} & =\left(a_{0}-a_{1}+-\ldots+(-1)^{N} a_{N}\right)^{2}= \\
& =\operatorname{sh}^{2}\left(v_{0}-2 v_{1}+2 v_{2}-+\ldots+(-1)^{N} 2 v_{N}-(-1)^{N} v_{N+1}\right), \\
B_{0} & =\left(b_{0}-b_{1}+-\ldots+(-1)^{N} b_{N}\right)^{2}= \\
& =\operatorname{ch}^{2}\left(v_{0}-2 v_{1}+2 v_{2}-+\ldots+(-1)^{N} 2 v_{N}-(-1)^{N} v_{N+1}\right),
\end{aligned}
$$

Последние две формулы согласуются с первой из формул (19).

Вместо выражений (16) и (17) для $A$ и $B$ бывают также полезны выражения вида 


$$
\begin{aligned}
& A=P_{0}+P_{1} \cos 2 \alpha+\ldots+P_{N} \cos 2 N \alpha, \\
& B=Q_{0}+Q_{1} \cos 2 \alpha+\ldots+Q_{N} \cos 2 N \alpha,
\end{aligned}
$$

причем, аналогично формулам (19),

$$
\begin{aligned}
& Q_{0}=1+P_{0}, \\
& Q_{k}=P_{k}, k=1,2, \ldots, N . \cdots
\end{aligned}
$$

Полагая в формулах (28) и (29) $\cos \alpha= \pm 1$, находим

$$
\begin{aligned}
& P_{0}+P_{1}+\ldots+P_{N}=A_{0}+A_{1}+\ldots+A_{N}, \\
& Q_{0}+Q_{1}+\ldots+Q_{N}=B_{0}+B_{1}+\ldots+B_{N},
\end{aligned}
$$

а полагая $\sin \alpha= \pm 1$, находим

$$
\begin{aligned}
& P_{0}-P_{1}+\ldots+(-1)^{N} P_{N}=A_{0} \\
& Q_{0}-Q_{1}+\ldots+(-1)^{N} Q_{N}=B_{0} .
\end{aligned}
$$

Отметим также равенства

$$
P_{N}=Q_{N}=2 a_{0} a_{N}=2 b_{0} b_{N}
$$

вытекающие из формул (13)-(15) и (28)-(30).

Перейдем к зависимости коэффициентов : $a_{0}, a_{1}, \ldots, a_{N}$ и $b_{0}, b_{1}, \ldots, b_{N}$ от $v_{01}, v_{1 \varsigma}, \ldots, v_{N, N+1}$. Она, вообще говоря, как видно из формул (3) и (4), довольнс: громоздка. Однако в явном виде ее достаточно знать только для $a_{0}, b_{0}, a_{N}$ и $b_{N}$ : Из формул $(3),(4),(14)$ и (15) находим

$$
\begin{aligned}
& a_{0}=\operatorname{ch} v_{01} \operatorname{ch} v_{12} \ldots \operatorname{ch} v_{N-1, N} \operatorname{sh} v_{N, N+1}, \\
& b_{0}=\operatorname{sh} v_{01} \operatorname{ch} v_{12} \ldots \operatorname{ch} v_{N-1, N} \operatorname{sh} v_{N, N+1}, \\
& a_{N}=\operatorname{sh} v_{01} \operatorname{ch} v_{12} \ldots \operatorname{ch} v_{N-1, N} \operatorname{ch} v_{N, N+1}, \\
& b_{N}=\operatorname{ch} v_{01} \operatorname{ch} v_{12} \ldots \operatorname{ch} v_{N-1, N} \operatorname{ch} v_{N, N+1} .
\end{aligned}
$$

Из:этих формул вытекает

$$
\begin{aligned}
\text { th } v_{01} & =a_{N} / b_{N}=b_{0} / a_{0}, \\
\text { th } v_{N, N+1} & =b_{0} / a_{N}=a_{0} / b_{N}
\end{aligned}
$$

H

$$
b_{0} b_{N}-a_{0} a_{N}=0 .
$$

Последняя формула согласуется с формулой (35).

Таковы основные исходные формулы. Процедура синтеза пленки на основе этих формул содержит три этапа: 1) задание коэффициентов $A_{0}, A_{1}, \ldots, A_{N}$ и $B_{0}, B_{1}, \ldots, B_{N}$ (будем в дальнейшем называть их для краткости большими коэффициентами); 2) нахождение коэффициентов $a_{0}, a_{1}, \ldots, a_{N}$ и $b_{0}, b_{1}, \ldots, b_{N}$ (будем их называть мальми коэффициентами); 3) нахождение показателей преломления слоев. Рассмотрим все три этапа по порядку.

\section{Задание больших коэффициентов}

Большие коэффициенты задаются так, чтобы спектральная кривая пленки возможно меньше отклонялась от заданной кривой. Простейшим 
и наиболее эффективным критерием служит минимум усредненного по спектру квадрата отклонения. Этот метод впервые был успешно применен в $\left[{ }^{6}\right]$ для синтеза просветляющих покрытий (см. также $\left[{ }^{5}\right]$, гл. VI). Пусть $S(\alpha)$ - заданная функция для $R / T$. Тогда названный критерий запишется в виде

$$
\frac{1}{\alpha_{2}-\alpha_{1}} \int_{\alpha_{1}}^{\alpha_{2}}\left[A_{0}+A_{1} \cos ^{2} \alpha+\ldots+A_{N} \cos ^{2 N} \alpha-S(\alpha)\right]^{2} d \alpha=\min ,
$$

где $\alpha_{1}, \alpha_{2}$ - границы того спектрального интервала, для которого пленка синтезируется. Конечно, вместо задания функции $S$ для $R / T$ можно задавать функцию $1+S$ для $T^{-1}$; так ккак $T^{-1}-(1+S)=$ $=R / T-S$, то условие (43) остается при этом неизменным.

Вычислив интеграл (43), минимизируем его с учетом дополнительного условия (22), где $v_{0}$ и $v_{N+1}$ имеют заданные значения. Это приводит к системе линейных уравнений для $A_{0}, A_{1}, \ldots, A_{N}$. Отсюда и определяются все эти коэффициенты. Формула (19) дает также коэффициенты $B_{0}, B_{1}, \ldots, B_{N}$.

Необходимо иметь в виду, что, каким бы образом ни задавались большие коэффициенты, они должны удовлетворять при всех вещественных значениях $\alpha$ (а не только, скажем, в интервале $\alpha_{1} \leqslant \alpha \leqslant \alpha_{2}$ ) неравенствам

и

$$
A_{0}+A_{1} \cos ^{2} \alpha+\ldots+A_{N} \cos ^{2 N} \alpha \geqslant 0
$$

$$
B_{0}+B_{1} \cos ^{2} \alpha+\ldots+B_{N} \cos ^{2 N} \alpha \geqslant 1,
$$

вытекающим из формул (13) и (16)-(18). Задавать большие коэффициенты с нарушением этих условий не имеет смысла, так как тогда нельзя было бы вычислить малые коэффициенты; такая задача синтеза просто не имела бы решения.

\section{Нахождение малых коэффициентов}

Зная большие коэффициенты, можно (при условии выполнения неравенств (44) и (45)) найти малые. Те и другие связаны соотношениями (см. формулы (13)-(17))

$$
\begin{gathered}
A_{0}+A_{1} \cos ^{2} \alpha+\ldots+A_{N} \cos ^{2 N} \alpha= \\
=\left(a_{0}+a_{1} e^{2 i \alpha}+\ldots+a_{N} e^{2 i N \alpha}\right)\left(a_{0}+a_{1} e^{-2 i \alpha}+\ldots+a_{N} e^{-2 i N \alpha}\right)
\end{gathered}
$$

и

$$
\begin{gathered}
B_{0}+B_{1} \cos ^{2} \alpha+\ldots+B_{N} \cos ^{2 N} \alpha= \\
=\left(b_{0}+b_{1} e^{2 i \alpha}+\ldots+b_{N} e^{2 i N \alpha}\right)\left(b_{0}+b_{1} e^{-2 i \alpha}+\ldots+b_{N} e^{-2 i N \alpha}\right) .
\end{gathered}
$$

Оба являются тождествами относительно $\alpha$, что и позволяет определить из них малые коэффициенты, когда известны большие. Вид формул (46) и (47) совершенно одинаков; формулы (20), (22), (24) и (26) тоже вполне аналогичны формулам (21), (23), (25) и (27). Отсюда следует, что нахождение обеих групп (т. е. $a_{k}$ и $b_{k}$ ) малых коэффициентов требует одного и того же алгоритма. Поэтому перепишем уравнения (46) и (47) в едином виде как

$$
\begin{gathered}
C_{a}+C_{1} \cos ^{2} \alpha+\ldots+C_{N} \cos ^{2 N} \alpha= \\
=\left(c_{0}+c_{1} e^{2 i \alpha}+\ldots+c_{N} e^{2 i N \alpha}\right)\left(c_{0}+c_{1} e^{-2 i \alpha}+\ldots+c_{N} e^{-2 i N \alpha}\right),
\end{gathered}
$$


где $C_{k}$ и $c_{k}$ означают или $A_{k}$ и $a_{k}$, или $B_{k}$ и $b_{k}$. Аналогично перепишем формулы $(20)-(27)$ в виде

$$
\begin{gathered}
c_{0}+c_{1}+\ldots+c_{N}=\mathrm{xh}\left(v_{0}-v_{N+1}\right) \\
C_{0}+C_{1}+\ldots+C_{N}=\left(c_{0}+c_{1}+\ldots+c_{N}\right)^{2}=\mathrm{xh}^{2}\left(v_{0}-v_{N+1}\right), \\
c_{0}-c_{1}+-\ldots+(-1)^{N} c_{N}= \\
=(-1)^{N} \text { xh }\left(v_{0}-2 v_{1}+2 v_{2}-+\ldots+(-1)^{N} 2 v_{N}-(-1)^{N} v_{N+1}\right)
\end{gathered}
$$

и

$$
\begin{gathered}
C_{0}=\left(c_{0}-c_{1}+-\ldots+(-1)^{N} c_{N}\right)^{2}= \\
=\operatorname{xh}^{2}\left(v_{0}-2 v_{1}+2 v_{2}-+\ldots+(-1)^{N} 2 v_{N}-(-1)^{N} v_{N+1}\right),
\end{gathered}
$$

где хһ означает sh, если $c_{k}, C_{k}$ суть $a_{k}, A_{k}$, и $\mathrm{ch}$, если $c_{k}, C_{k}$ суть $b_{l}, B_{k}$.

Итак, требуется, зная $C_{0}, C_{1}, \ldots, C_{N}$, найти из уравнения (48) $c_{0}, c_{1}, \ldots, c_{N}$ (с учетом дополнительного условия (49)). Для этого предложим следующий алгоритм.

Рассматривая $\alpha$ как комплексный аргумент, введем величины

$$
z=\cos ^{2} \alpha
$$

и

так что

$$
u^{ \pm}=2 z-1 \pm 2 i \sqrt{z(1-z)}
$$

$$
u^{ \pm}=e^{ \pm 2 i \alpha}
$$

и

$$
z=\left(u^{ \pm}+1\right)^{2} / 4 u^{ \pm}
$$

Тогда, обозначая

и

$$
\Phi(z) \equiv C_{0}+C_{1} z+\ldots+C_{N} z^{N}
$$

$$
\varphi(u) \equiv c_{0}+c_{1} u+\ldots+c_{N} u^{N},
$$

можем переписать уравнение (48) в виде

$$
\Phi(z)=\varphi\left(u^{+}\right) \varphi\left(u^{-}\right)
$$

Подчеркнем, что это равенство является тождеством относительно $\alpha$, т. е. оно верно не только для вещественных, но и для комплексных значений $\alpha$. Далее найдем нули полинома $\Phi(z)$, т. е. корни уравнения $N$-й степени

$$
C_{0}+C_{1} z+\ldots+C_{N} z^{N}=0 .
$$

Обозначим корни через $z_{1}, z_{2}, \ldots, z_{N}$. Соответствуюшие им по формуле (54) значения $u^{ \pm}$обозначим через $u_{1}^{ \pm}, u_{2}^{ \pm}, \ldots, u_{N}^{ \pm}$. Из формулы (59) следует, что $\varphi\left(u_{k}^{+}\right)=0$ или $\varphi\left(u_{k}^{-}\right)=0(k=1,2, \ldots, N)$. Учитывая вдобавок условие (49), получим для $c_{0}, c_{1}, \ldots, c_{N}$ систему линейных уравнений

$$
\begin{gathered}
c_{0}+c_{1}+c_{2}+\ldots+c_{N}=\mathrm{xh}\left(v_{0}-v_{N+1}\right) \\
c_{0}+c_{1} u_{1}+c_{2} u_{1}^{2}+\ldots+c_{N} u_{1}^{N}=0 \\
c_{0}+c_{1} u_{2}+c_{2} u_{2}^{2}+\ldots+c_{N} u_{2}^{N}=0 \\
\vdots \\
c_{0}+c_{1} u_{N}+c_{2} u_{N}^{2}+\ldots+c_{N} u_{N}^{N}=0
\end{gathered}
$$


в которой каждая из величин $u_{h}(k=1,2, \ldots, N)$ может означать либо $u_{k}{ }^{+}$, либо $u_{k}{ }^{-}$. Такнм образом, эта система может быть напнсана в различных вариантах. Не все варианты, однако, допустимы, так как $c_{0}, c_{1}, \ldots, c_{N}$ должны быть вещественны, а величнны $u_{h}^{ \pm}$, вообще говоря, комплексны.

Если все корни уравнения (60) различны, то различны и все $u_{k}$, в силу чего система (61) содержит $N+1$ независимых уравнений и имеет отличный от нуля определитель. Следовательно, из нее можно найти искомые малые коэффициенты. Если же уравнение (60) имеет кратные корни, то система (61) должна быть видоизменена. В нижеследующей покажем, как это делается, и подсчитаем число вариантов, гарантирующих вещественность $c_{0}, c_{1}, \ldots, c_{N}$.

Целесообразно различать четыре рода корней уравнения (60).

Пусть, во-первых, $z$ - вещественный корень, удовлетворяющий неравенству $z<0$ или $z>1$ (индекс корня здесь опускаем). Тогда, 'согласно формуле (54), $u^{+}$и $u^{-}$вещественны-и различны. Обозначим кратность корня через p. В систему линейных уравнений дли $c_{0}, c_{1}, \ldots, c_{N}$ этот корень должен дать $p$ независимых уравнений: Их получим в $p+1$ вариантах следующим образом. Будем рассматривать $u^{+}$как группу бесконечно близких значений, числом $m$, и $u^{-}$тоже как группу бесконечно близких значений, числом $p-m$. Тогда получим следующие уравнения:

$$
\begin{aligned}
& \varphi\left(u^{+}\right)=0, \\
& \varphi^{\prime}\left(u^{+}\right)=0, \\
& \vdots \\
& \varphi^{(m-1)}\left(u^{+}\right)=0, \\
& \varphi\left(u^{-}\right)=0, \\
& \varphi^{\prime}\left(u^{-}\right)=0, \\
& \vdots \\
& \varphi^{(p-m-1)}\left(u^{-}\right)=0,
\end{aligned}
$$

где производные берутся по аргументу $u^{+}$или $u^{-}$, а под $\varphi(u)$ следует понимать линейную относительно $c_{0}, c_{1}, \ldots, c_{N}$ форму, согласно формуле (58). Уравнения (62), числом $p$, линейно независимы, имеют вещественные коэффициенты и могут быть написаны в $p+1$ вариантах, соответственно $p+1$ значениям $m=0,1, \ldots, p$. *

Во-вторых, пусть $z$ - комплексный корень (с отличной от нуля мнимой частью). Тогда корнем уравнения (60) является также $z^{*}$. Пусть эта пара комплексно-сопряженных корней имеет кратность $p$. Тогда, согласно формуле $(54), u^{+}$и $u^{-}$комплексны й различны, причем $u^{+}\left(z^{*}\right)=u^{-*}(z)$ и $u^{-}\left(z^{*}\right)=u^{+^{*}}(z)$. В систему уравнений для $c_{0}, c_{1}, \ldots$ $\ldots, c_{N}$ пара корней $z, z^{*}$ должна дать $2 p$ независимых уравненйй, имеющих попарно комплексно-сопряженные коэффициенты (это необходимо для вещественности $\left.c_{0}, c_{1}, \ldots, c_{N}\right)$. Аналогично уравнениям (62), эту группу уравнений можно написать в $p+1$ вариантах:

$$
\begin{array}{ll}
\varphi\left(u^{+}(z)\right)=0, & \varphi\left(u^{-}\left(z^{*}\right)\right)=0, \\
\varphi^{\prime}\left(u^{+}(z)\right)=0, & \varphi^{\prime}\left(u^{-}\left(z^{*}\right)\right)=0,
\end{array}
$$

* В [ $\left.{ }^{4}\right]$ при аналогичном подсчете ошнбочно учтены төлько два варианта, соответствующие значаниям $m=0$ и $m=p$. 


$\begin{array}{cc}\vdots & \vdots \\ \varphi^{(m-1)}\left(u^{+}(z)\right)=0, & \varphi^{(m-1)}\left(u^{-}\left(z^{*}\right)\right)=0, \\ \varphi(u-(z))=0, & \varphi\left(u^{+}\left(z^{*}\right)\right)=0, \\ \varphi^{\prime}\left(u^{-}(z)\right)=0, & \varphi^{\prime}\left(u^{+}\left(z^{*}\right)\right)=0, \\ \vdots & \vdots \\ \varphi^{(p-m-1)}\left(u^{-}(z)\right)=0, & \varphi^{(p-m-1)}\left(u^{+}\left(z^{*}\right)\right),\end{array}$

где $m=0,1, \ldots, p$. Уравнения, написанные в правом"столбце, имеют коэффициенты, комплексно-сопряженные коэффициентам уравнений, написанных в левом столбце.

Третьего рода корни уравнения (60) вещественны и заключены в. интервале $0<z<1$. В этом случае $u^{+}$и $u^{-}$различны и комплексносопряженны друг другу, а $\alpha$ вещественно, прнчем $\alpha \neq 0, \alpha \neq \pi / 2$. Заметим, что, согласно неравєнствам (44) и (45), корни этого рода возможны только піри $C \equiv A$ и только с четной кратностью: Обозначал кратность опять через $p$, мы могли бы написать $p$ уравнений для $c_{0}, c_{1}, \ldots, c_{N}$ такого же вида, как в $(62)$. Однако вместо $p+1$ вариантов имеем теперь только один, именно при $m=p / 2$., Только в этом варианте уравнения имеют попарно комплексно-сопряженные коэффициенты. Итак,

$$
\begin{array}{cc}
\varphi\left(u^{+}\right)=0, & \varphi\left(u^{-}\right)=0, \\
\varphi^{\prime}\left(u^{+}\right)=0, & \varphi^{\prime}\left(u^{-}\right)=0, \\
\vdots & \vdots \\
\varphi^{(p / 2-1)}\left(u^{+}\right)=0, & \varphi^{(p / 2-1)}\left(u^{-}\right)=0 .
\end{array}
$$

$\mathrm{K}$ четвертому роду корней относятся значения $z=0$ и $z=1$. В: этих случаях $u^{+}=u^{-}=-1$ или $u^{+}=u^{-}=1$ соответственно, тогда как $\alpha \stackrel{=}{=}(n+1 / 2) \pi$ или $\alpha=n \pi$ ( $n$ целое). Корни этого рода возможны, как и корни третьего рода, только при $C \equiv A$, но их кратность не обязательно четна. Обозначая кратность чепез $p$, получим, очевидно, единственный вариант $p$ уравнений:

$$
\begin{gathered}
\varphi(u)=0, \\
\varphi^{\prime}(u)=0, \\
\vdots \\
\varphi^{(p-1)}(u)=0 .
\end{gathered}
$$

(А Резюмируя, приходим к следующему выводу. В любом случае корни уравнения (60) дают систему $N$ линейных однсродных линейно независимых уравнений для $c_{0}, c_{1}, \ldots, c_{N}$. Вместе с уравнением (49) получается определяющая эти малые коэффициенты система $N+1$ уравнений. Она может быть составлена в нескольких варнантах. Число вариантов зависит от кратностей корней уравнения (60) первсго и второго родов, т. е. вещественных корней, лежащих вне интервала $(0,1)$, и комплексных корней. Именно; полное число $P_{c}$ вариантов равно

$$
P_{c}=\prod_{i}\left(p_{i}+1\right)
$$

где $i$ нумерует все различные корни первого рода и все различные пары комғлексно-сопряженных корней второго рода. Если, в частности, все корни имеют единичную кратность, то 


$$
P_{c}=2^{r+q},
$$

где $r$ - число корней первого рода и $q$ - число пар корней второго рода.

Необходимо следующее дополнительное разъяснение. Если уравнение $(60)$ имеет корень четвертого рода $z=1$, то одним из уравнений системы (65) будет уравнение $c_{0}+c_{1}+\ldots+c_{N}=0$. Оно согласуется с уравнением (49) только тогда, если $v_{0}=v_{N+1}$ (в противном случае, стало быть, корня $z=1$ быть не может), и только при $\mathrm{xh}=\mathrm{sh}$. Здесь мы вновь убеждаемся, что корень $z=1$ возможен только при $C \equiv A$. Но в данном случае уравнение (49) не может быть добавлено к системе как независимое. Система уравнений для $a_{0}, a_{1}, \ldots, a_{N}$ содержит, следовательно, только $N$ однородных уравнений. Ею определяются только отношения коэффициентов $a_{0}, a_{1}, \ldots, a_{N}$, но не они сами. Для однозначного определения этих коэффициентов следует воспользоваться соотношением (42), где $b_{0}$ и $b_{N}$ должны быть уже известны. Если же корня $z=1$ нет, то это соотношение может служить для контроля. Формула (52) тоже является контрольной.

Так как обе группы малых коэффициентов, $a_{k}$ и $b_{k}$, вычисляются независимо друг.от друга, то полное число вариантов их значений равно

$$
P=P_{a} P_{b}
$$

где $P_{a}$ и $P_{b}$ определяются по формуле $(66)$.

\section{Нахождение показателей преломления слоев}

После того, как малые коэффициенты найдены, по ним определяются показатели преломления слоев следующим образом.

Будем рассматривать наряду с полной $N$-слойной пленкой последовательность неполных $(N-m)$-слойных пленок $(m=1,2, \ldots, N-1)$, в которых исходной средой является $m$-й слой, a $(m+1)$-й, $(m+2)$-й, $\ldots, \quad N$-й слои те же, что и у полной пленки. Обозначим матрицу $F$ полной пленки (см. формулу $(8))$ через $F^{(0)}$, а матрицу $(N-m)$-слойной пленки через $F^{(m)}$. Тогда

и

$$
F^{(m)}=G\left(v_{m}\right) L_{m+1} L_{m+2} \ldots L_{N} G\left(-v_{N+1}\right)
$$

$$
F^{(m+1)}=G\left(v_{m+1}\right) L_{m+2} L_{m+3} \ldots L_{N} G\left(-v_{N+1}\right) .
$$

Из этих двух формул следует

$$
F^{(m+1)}=G\left(v_{m+1}\right) L_{m+1}^{-1} G\left(-v_{m}\right) F^{(m)} .
$$

Но, согласно формулам (9) и (10),

$$
G\left(v_{m+1}\right) L_{m+1}^{-1} G\left(-v_{m}\right)=\left(\begin{array}{c}
e^{-i \alpha} \operatorname{ch} v_{m, m+1}-e^{-i \alpha} \operatorname{sh} v_{m, m+1} \\
-e^{i \alpha} \operatorname{sh} v_{m, m+1} e^{i \alpha} \operatorname{ch} v_{m, m+1}
\end{array}\right)
$$

подставляя это выражение в формулу (71) и учитывая формулу (12), находим:

$$
\begin{aligned}
& b^{(m+1)}=e^{-i \alpha}\left(\operatorname{ch} v_{m, m+1} b^{(m)}-\operatorname{sh} v_{m, m+1} a^{(m)}\right), \\
& a^{(m+1)}=e^{i \alpha}\left(-\operatorname{sh} v_{m, m+1} b^{(m)}+\operatorname{ch} v_{m, m+1} a^{(m)}\right) .
\end{aligned}
$$

Далее, аналогично формулам (14) и (15), напишем формулы такого же вида для неполных пленок с индексами $m$ и $m+1$ : 


$$
\begin{aligned}
& b^{(m+1)}=e^{-i(N-m-1) \alpha}\left(b_{0}^{(m+1)}+b_{1}^{(m+1)} e^{2 i \alpha}+\ldots+b_{N-m-1}^{(m+1)} e^{2 i(N-m-1) \alpha}\right), \\
& a^{(m+1)}=e^{-i(N-m-1) \alpha}\left(a_{0}^{(m+1)}+a_{1}^{(m+1)} e^{2 i \alpha}+\ldots+a_{N-m-1}^{(m+1)} e^{2 i(N-m-1) \alpha}\right)
\end{aligned}
$$

и

$$
\begin{aligned}
& b^{(m)}=e^{-i(N-m) \alpha}\left(b_{0}^{(m)}+b_{1}^{(m)} e^{2 i \alpha}+\ldots+b_{N-m}^{(m)} e^{2 i(N-m) \alpha}\right), \\
& a^{(m)}=e^{-i(N-m) \alpha}\left(a_{0}^{(m)}+a_{1}^{(m)} e^{2 i \alpha}+\ldots+a_{N-m}^{(m)} e^{2 i(N-m) \alpha}\right) .
\end{aligned}
$$

Подставляя эти выражения в формулы (73) и учитывая, что, аналогично формуле (40),

$$
\text { th } v_{m, m+1}=a_{N-m}^{(m)} / b_{N-m}^{(m)}=b_{0}^{(m)} / a_{0}^{(m)},
$$

находим:

$$
\begin{array}{r}
b_{k}^{(m+1)}=b_{k+1}^{(m)} \operatorname{ch} v_{m, m+1}-a_{k+1}^{(m)} \operatorname{sh} v_{m, m+1}, \\
a_{k}^{(m+1)}=a_{k}^{(m)} \operatorname{ch} v_{m, m+1}-b_{k}^{(m)} \operatorname{sh} v_{m, m+1}, \\
m=0,1, \ldots, N-1, \\
k=0,1, \ldots, N-m-1 .
\end{array}
$$

Теперь, зная малые коэффициенты $b_{k}{ }^{(0)}$ и $a_{k}{ }^{(0)}$ для полной пленки, вычисленные способом, изложенным в предыдуціем разделе, можем вычислить последовательно все величины $v_{01}, v_{12}, \ldots, v_{N, N+1}$. Сначала находим по формуле (40) $v_{01}$. Затем, полагая в формулах (77) $m=0$, вычисляем малые коэффициенты $b_{k}^{(1)}$ и $a_{k}^{(1)}$. После этого формула (76) с $m=1$ дает $v_{12}$. Беря далее в формулах (77) $m=1$, вычисляем все малые коэффициенты $b_{k}{ }^{(2)}$ и $a_{k}{ }^{(2)}$ и т. д. Таким образом, применяя попеременно формулы (76) и (77), находим всю последовательность величин $v_{01}, v_{12}, \ldots, v_{N, N+1}$. Отсюда по формуле (7) вычисляем показатели преломления всех слоев. На этом синтез заканчивается.

В заключение отметим, что изложенный метод может дать нереальные - слишком большие или слишком малые - значения показателей преломления. Однако, располагая несколькими вариантами, мы всегда можем выбрать наилучший из них. Если и он оказывается неудовлетворительным, то остается только повторить синтез, исходя из иных значений больших коэффициентов. Для этого следует жесткое условие минимума (43) заменить более слабым условием, гарантирующим всетаки достаточную близость спектральной кривой к требуемой.

\section{Л И ТЕ Р А Т У Р А}

1. С ос с и Л., Изв. АН ЭССР, Физ. Матем., 23, № 3, 229-237 (1974).

2. С осс и Л., Изв. АН ЭССР, Физ. Матем., 26, № 1, 28-36 (1977).

3. P e g i s, R. J., J. Opt. Soc. America, 51, № 11, 1255-1264 (1961).

4. К а рд П., Ихе р Х., Изв. АН ЭССР, Физ. Матем., 26, № 1, 13-27 (1977).

5. К а рд П. Г., Анализ и синтез многослойных интерференционных пленок, Таллин, «Валгус», 1971.

6. К а р д П. Г., Оптика и спектроскопия, 2, вып, 2, 245-253 (1957).

Тартуский государственный университет
Поступнла в редакцию 2/IV 1979 


\section{P. KARD}

\section{VORDPAKSUSTE KIHTIDEGA DIELEKTRILISTE INTERFERENTSKILEDE SONTEESI TEOORIAST}

Võrdpaksuste kihtidega dielektrilise interferentskile sünteesi eesmärk on etteantud kihtide arvu $N$ ja piiravate keskkondade murdumisnäitajate $n_{0}, n_{N+1}$ korral määrata kihtide murdumisnäitajad $n_{1}, n_{2}, \ldots, n_{N}$ nii, et kile spektraalkõver $R / T$, kus $R$. ja $T$ on kile peegeldumis- ja läbilaskvustegur, erineks vơimalikult vähe etteantud funktsioonist $S(\alpha)$, kus $\alpha=(2 \pi / \lambda) n h$ ( $\lambda$ on lainepikkus ja $n h$ iga kihi optiline paksus). Lähtevalemid on (13)-(17), kus $A=R / T$ ja $B=T^{-1}$, kuna «suured» kordajad $A_{k}, B_{k}$ ja «väikesed» kordajad $a_{k}, b_{k}$ sōltuvad kindlal viisil kihtide ja piiravate keskkondade murdumisnäitajatest. Süntees toimub kolmes järgus. Esmalt määratakse miinimumitingimusest (43) suured kordajad. Teises järgus leitakse väikesed kordajad. Selleks tuleb lahendada võrrand (60), vōttes kord $C_{k} \equiv A_{k}$ ja seejärél $C_{k} \equiv B_{k}$. Kui kõik $N$ lahendit on erinevad, siis määrab väikesed kordajad (vastavalt kas $a_{h}$ või $b_{k}$ ) vōrrandisüsteem (61), kus $u_{k}$ on lahendile $z_{k}$ valemi (54) järgi vastav suurus ning $\mathrm{xh}\left(v_{0}-v_{N+1}\right)=$ $=\frac{n_{0}-n_{N+1}}{2\left(n_{0} n_{N+1}\right)^{1 / 2}} \quad c_{k} \equiv a_{h} \quad$ puhul $\quad$ ja $\quad x h\left(v_{0}-v_{N+1}\right)=\frac{n_{0}+n_{N+1}}{2\left(n_{0} n_{N+1}\right)^{1 / 2}} \quad c_{k} \equiv b_{k}$

puhul. Kui võrrandil $(60)$ on kordseid lahendeid, siis saab vōrrandisüsteem teistsuguse kuju. Kolmas järk seisneb murdumisnäitajate leidmises väikeste kordajate järgi. Selleks on rekurrentsed valemid (76) ja (77), mida tuleb kasutada vaheldumisi, alustades valemist (76) $m=0$ juures ning loppetades sama valemiga $m=N$ juures. Sutirused $v_{01}$. $v_{12}, \ldots, v_{N, N+1}$ määravad valemi (7) järgi kōik otsitavad murdumisnäitajad.

\section{P. KARD}

\section{ON THE THEORETICAL DESIGN OF THE DIELECTRIC INTERFERENCE FILMS WITH EQUAL OPTICAL THICKNESSES OF LAYERS}

Given the number, $N$, of the layers and the refractive indices, $n_{0}, n_{N+1}$, of the ambient media, a dielectric interference film. with equal optical thicknesses of layers is to be designed, having a given spectral reflectance, $R$, and transmittance, $T$. The basic formulae for this purpose are (13)-(17), where $A=R / T, B=T^{-1}$ and $\alpha=(2 \pi / \lambda) n \hbar, \lambda$ being wavelength and $n h$ optical thickness of each layer. The egreat» coeificients $A_{k}, B_{k}$ and the «small» coefficients $a_{k}, b_{k}$ depend in a certain manner on the refractive indices of the layers and the ambient media. The design consists of three stages. Firstly one has to determine the great coefficients. This is done by the minimum condition (43), ensuring the best fitting of the spectral characteristic $R / T$ to a given function $S(\alpha)$. In the second stage the small coefficients must be found. For this purpose the equation (60), both for $C_{k} \equiv A_{h}$ and $C_{k} \equiv B_{k}$, must be solved. If all $N$ roots $z_{k}$ of this equation are different, then the small coefficients can be found from the system of linear equations (61), where: $u_{k}$ 's depend on $z_{k}$ 's via the formula (54), and $\operatorname{xh}\left(v_{0}-v_{N+1}\right)=\frac{n_{0}-n_{N+1}}{2\left(n_{0} n_{N+1}\right)^{1 / 2}}$, when. $c_{k} \equiv a_{k}, \quad$ and $\quad x h\left(v_{0}-v_{N+1}\right)=\frac{n_{0}+n_{N+1}}{2\left(n_{0} n_{N+1}\right)^{1 / 2}}$,

when $c_{k} \equiv b_{k}$. If not all roots of the equation $(60)$ are different, then the system $(61)$ must be modified. In the third stage the refractive indices of the layers are computed from the small coefficients. For this serve recurrent formulae (76) and (77), which must be alternately used, beginning with the formula (76) with $m=0$ and finishing with the same formula with $m=N$. As a result one obtains the quantities $v_{01}, v_{12}, \ldots$. $v_{N}, N+1$, from which refractive indices of all layers can be computed via formula $(7)$. 